
\title{
3 Research buare \\ " An Efficient One Pot Multicomponent Synthesis of Dihydro Pyrazolyl Bithiazole Derivatives as Potential Anticancer Agents and Their Molecular Docking Studies"
}

\section{Anjaneyulu V}

Osmania University University College of Science

KRISHNAIAH VAARLA

National Institute of Technology Warangal

RajeshKumar Kesharwani

Nehru Gram Bharati University

Leelavathi Panaganti ( $\sim$ leelaou@gmail.com )

Osmania University University College of Science

\section{Research Article}

Keywords: Multicomponent reaction, dihydropyrazolyl bithiazoles, anticancer activity and molecular docking studies

Posted Date: January 19th, 2022

DOI: https://doi.org/10.21203/rs.3.rs-1243512/v1

License: (c) (1) This work is licensed under a Creative Commons Attribution 4.0 International License.

Read Full License 


\section{Abstract}

A novel series of dihydropyrazolyl bithiazoles were efficiently synthesized through simple reaction conditions via one pot multicomponent reaction of 2-bromo-1-(4-methyl-2-phenyl thiazol-5-yl)ethan-1-one (1), thiosemicarbazide (2) and chalcones ( $3 a-k)$ in the presence of sodium hydroxide in ethanol under reflux condition. The reaction generates two potential five membered heterocylic pharmacophores i.e. Hantzsch thiazole and dihydropyrazole in one step through operational simplicity, shorter reaction time with high yields. The newly synthesized compounds were well characterized by $I R,{ }^{1} \mathrm{H},{ }^{13} \mathrm{C}$ NMR and Mass spectral data. All the newly synthesized compounds were evaluated for their anticancer activity against human cancer cell lines and calculated their binding energy values with respect to 3ert protein. The compounds $4 \mathrm{f}, 4 \mathrm{~g}$, $4 \mathrm{~h}$ and $4 \mathrm{j}$ exhibited excellent activity against MCF-7 cancer cell line with $\mathrm{IC}_{50}$ Values of $3.47 \pm 0.28,1.36 \pm 0.74,3.76 \pm 0.35$ and $4.14 \pm 0.26 \mu \mathrm{M}$ concentrations and studied molecular interaction of probable target protein human Estrogen Receptor Alpha protein (3ert.pdb) using docking simulation.

\section{Introduction}

The main aim of modern drug discovery is to synthesize therapeutically active compounds by using simple reaction pathways. Multicomponent reactions (MCRs) are one of the versatile chemical transformations and useful tool for the synthesis of structurally diversified more complex therapeutic active molecules from readily available starting materials in a single step process. Due to the selectivity, efficiency in terms of reduction of number of steps, no waste generation, atom economy, high yields, less reaction time and eco compatibility, Multicomponent reactions are considered as ideal green and powerful synthetic methods in organic synthesis [1-4] and drug discovery $[5,6]$.

Heterocycles are the most important pharmacophores in medicinal chemistry and researchers are aiming to develop the efficient synthetic methods to obtain pharmaceutically active heterocycles. Heterocycles also contributed to the improvement of quality of life through their applications in the field of biomolecules [7 - 9], agrochemicals [10 - 12], materials [13], food and cosmetics [14, 15].

Thiazoles and dihydropyrazoles are versatile five membered heterocyclic compounds, which are widely used in the field of medicinal chemistry, natural products and functional materials. Most of the thiazoles have been reported to exhibit vast pharmacological applications including antimicrobial [16,17], antivira [18 - 20], antitubercular [21, 22], anticancer [23 - 26], anti-inflammatory [27 - 29], antitrypanosomal [30, 31] and anti fungal activity [32,33]. Pyrazoles represent another important nitrogen containing heterocycles that have attracted much more attention among the medicinal chemists due to their extensive applications in the field of drug discovery [34 - 40], agrochemicals [41] and materials [42 - 45]. Moreover the thiazole and pyrazole heterocycles are used as starting materials to construct the diversified heterocyclic systems and also they constitute an interesting template for combinatorial chemistry [46-49]. Some of the therapeutic active dihydropyrazole and thiazole heterocycles were given Figure 1. 
In view of the biological significance of thiazoles and dihydropyrazoles, in continuation of our interest in synthesis of biologically active heterocyclic compounds, we felt that it is worthwhile to explore the synthesis and scope of thiazoles and dihydropyrazoles. Here in, we wish to report the development of new thiazole and dihydropyrazole heterocyclic compounds in a single molecular frame work through simple and efficient one pot multicomponent chemical transformation of 2-bromo-1-(4-methyl-2-phenyl thiazol-5-yl)ethan-1-one (1), thiosemicarbazide (2) and various substituted chalcones (3 a-k) and evaluation of their anticancer activity properties. At present anticancer activity study and molecular docking studies play a crucial role in order to understand the molecular level interaction and active site residues property in relation to biological activity.

\section{Experimental}

The organic solvents and the reagents like thiosemicarbazide, sodium hydroxide were procured from commercial sources and used as such without any further purification. The reactions were monitored through thin layer chromatography using pre coated silica gel plates and the spots were visualized under UV light and lodine vapours. Melting points were recorded in open capillary melting tube using Stuart melting point apparatus (SMP-30) and are uncorrected. Infra red spectra were recorded on Perkin-Elmer (Spectrum -100S) spectrophotometer. The ${ }^{1} \mathrm{H}$ and ${ }^{13} \mathrm{C}$ were analyzed by using Bruker spectrometer at 400 and $100 \mathrm{MHz}$ using tetramethylsilane (TMS) as internal standard. The mass spectra were recorded on Water micromass API instrument.

\section{Anticancer activity evaluation of 2-(5-(Aryl)-3-heteryl-4,5-dihydro-1 H-pyrazol-1-yl)-4'-methyl-2'-phenyl-4,5'- bithiazole derivatives (4a-k) by using standard MTT assay:}

The novel 2-(5-(Aryl)-3-heteryl-4,5-dihydro-1 H-pyrazol-1-yl)-4'-methyl-2'-phenyl-4,5'-bithiazole derivatives $(4 a-k)$ were evaluated for their anticancer activity property against a panel of four human cancer cell lines like Non-small-cell lung cancer cell lines (A549), prostate cancer (DU-145), breast cancer (MCF-7) and Human neuroblastoma (SKNSH) cell lines by using standard MTT protocol in a 96 well plate. The four cancer cell lines were procured from American Type Culture Collection (ATCC) and cells were grown in Dulbecco's modified Eagle medium (DMEM) with 10\% fatal bovine serum (FBS), 1\% antibiotics and incubated at $37^{\circ} \mathrm{C}$ in a humidified incubator with $5 \% \mathrm{CO}_{2}$. The newly synthesized compounds (4a-k) were dissolved in cell culture grade dimethyl sulphoxide solvent. The four cancerous cell lines were taken in a 96 well tissue culture plate $\left(10^{4}\right.$ cells/ each well) and the cells were treated with different concentrations of synthesized compounds and incubated for about $48 \mathrm{~h}$. After $48 \mathrm{~h}$ of incubation, the medium was removed, washed with Dulbecco's phosphate buffer saline (DPBS) and treated with $10 \mu \mathrm{L}$ of 3-(4,5dimethylthiazol-2-yl)-2,5-diphenyl tetrazolium bromide (MTT) solution in $200 \mu \mathrm{L}$ of culture medium. The MTT solution treated cells were incubated for $1 \mathrm{~h}$ and absorbance was measured at $562 \mathrm{~nm}$ using spectrophotometer. The absorbance of the treated cells was compared with the control. The MTT assay was performed in triplicate and the results were shown as percentage reduction in cell viability.

\section{In silico Study:}




\section{Preparation of Target and Ligands:}

Based on available literature and scientific fact, we have selected Estrogen Receptor Alpha (Era) protein as a probable target against breast cancer (MCF-7). The 3-D structure of Human Estrogen Receptor Alpha protein (3ERT.PDB) [54] with co-crystalized bound ligand 4-Hydroxytamoxifen and 79 water molecules was downloaded from Protein Data Bank. The target protein was prepared in the form of .pdbqt format file using MGLTool by removing bound ligand and water molecules. The selected ligands (Dihydro Pyrazolyl Bithiazole Derivatives) were drawn using ChemSketch and saved in sdf format. These ligands were converted in pdb format using Open babel and was prepared in the form of .pdbqt format by using MGLTool by assigning maximum rotatable bonds. Hardware used for the current study having configuration HP Intel(R) Core(TM) i3, memory (RAM) $4.00 \mathrm{~GB}$, with 64-bit Operating system and $x 64$ based processor.

\section{Grid parameter and Docking Simulation:}

For the docking simulation study, active site was selected based on the bound ligand (OHT) and the grid parameter assigned with number of points in $X=100 ; Y=100 ; Z=100$ and center_X $=30.708$; center_y $=$ -1.057; center_z $=27.065 \AA$ and default grid spacing $(0.375 \AA)$ on target molecule. With the help of molecular docking simulation approach using AutoDock Vina (ADT Vina), the evaluation of selected ligands was performed against the target (3ERT.PDB), to determine the structure activity relationship (SAR). The representation of docked confirmation with target was generated in the form of 2-D and 3-D using LigPlus [55] and Molegro Molecular Viewer [56] respectively.

General Procedure for the synthesis of 2-(5-(Aryl)-3-heteryl-4,5-dihydro-1H-pyrazol-1-yl)-4'-methyl-2'-phenyl4,5'-bithiazole derivatives (4a-k):

A reaction mixture of 2-bromo-1-(4-methyl-2-phenylthiazol-5-yl)ethan-1-one (1, $1 \mathrm{mmol})$, thiosemicarbazide $(2,1 \mathrm{mmol})$, chalcones $(3 \mathrm{a}-\mathrm{k}, 1 \mathrm{mmol})$ and sodium hydroxide $(1.5 \mathrm{mmol})$ in ethanol $(5 \mathrm{~mL})$ were heated to $70^{\circ} \mathrm{C}$ for about $4-5 \mathrm{~h}$. The progress of the reaction was monitored by TLC. After completion of the reaction, the reaction mixture was cooled to room temperature and the separated solid was filtered. The solid obtained was recrystallized from ethanol to give the desired compound.

1. 4'-methyl-2'-phenyl-2-(5-phenyl-3-(thiophen-2-yl)-4,5-dihydro-1H-pyrazol-1-yl)-4,5'-bithiazole (4a):

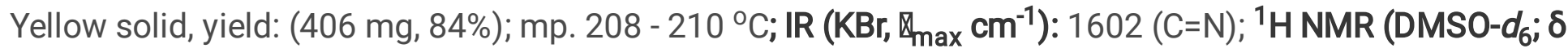
ppm): 2.48 (s, 3H), $3.32-3.40$ (dd, $J=23.2 \mathrm{~Hz}, 9.2 \mathrm{~Hz}, 1 \mathrm{H}), 3.93-4.03(\mathrm{dd}, J=23.2 \mathrm{~Hz}, 16 \mathrm{~Hz}, 1 \mathrm{H}), 5.59$

- $5.65(\mathrm{dd}, J=16 \mathrm{~Hz}, 9.2 \mathrm{~Hz}, 1 \mathrm{H}), 6.69(\mathrm{~s}, 1 \mathrm{H}, \mathrm{Ar}-\mathrm{H}), 7.09(\mathrm{t}, \mathrm{J}=6.8 \mathrm{~Hz}, 1 \mathrm{H}), 7.25-7.36(\mathrm{~m}, 2 \mathrm{H}, \mathrm{Ar}-\mathrm{H}), 7.41$

- $7.47(\mathrm{~m}, 7 \mathrm{H}, \mathrm{Ar}-\mathrm{H}), 7.60(\mathrm{~s}, 1 \mathrm{H}, \mathrm{Ar}-\mathrm{H}), 7.86-7.89(\mathrm{~m}, 2 \mathrm{H}, \mathrm{Ar}-\mathrm{H}) ;{ }^{13} \mathrm{C} \mathrm{NMR}\left(\mathrm{DMSO}-d_{6}+\mathrm{CDCl}_{3} ; \delta \mathrm{ppm}\right)$ : 
17.34, 44.31, 64.78, 105.65, 125.55, 126.12, 126.84, 127.91, 128.80, 129.13, 130.02, 133.49, 134.44, 141.42, 143.37, 149.27, 163.58, 164.23; MS (ESI m/z): $485.09[\mathrm{M}+\mathrm{H}]^{+}$.

2. 2-(5-(4-fluorophenyl)-3-(thiophen-2-yl)-4,5-dihydro-1H-pyrazol-1-yl)-4'-methyl -2'-phenyl-4,5'-bithiazole (4b)

Orange solid, yield: (446 mg, 89\%); mp. 185- 187 C; IR (KBr, $\mathbb{X}_{\max }$ cm$\left.^{-1}\right)$ : 1612 (C=N); ${ }^{1} \mathrm{H}$ NMR (DMSO- $\boldsymbol{d}_{6} ; \delta$ ppm): 2.51 (s, 3H), $3.29-3.34$ (dd, J=12.8 Hz, $6 \mathrm{~Hz}, 1 \mathrm{H}), 3.97-4.04$ (dd, J = 16.8 Hz, 4.8 Hz, $1 \mathrm{H}), 5.61-$ 5.66 (dd, $J=11.2 \mathrm{~Hz}, 5.6 \mathrm{~Hz}, 1 \mathrm{H}), 6.76$ (d, J = 4.8 Hz, 1H, Ar-H), 7.07 - 7.11 (m, 3H, Ar-H), 7.29 (s, 1H), 7.44 - $7.52(\mathrm{~m}, 5 \mathrm{H}, \mathrm{Ar}-\mathrm{H}), 7.74$ (d, J = $8.8 \mathrm{~Hz}, 1 \mathrm{H}, \mathrm{Ar}-\mathrm{H}), 7.90(\mathrm{~s}, 2 \mathrm{H}, \mathrm{Ar}-\mathrm{H}) ;{ }^{13} \mathrm{C} \mathrm{NMR}$ (DMSO- $d_{6}+\mathrm{CDCl}_{3} ; \delta$ ppm): 17.06, 44.24, 64.16, 105.87, 115.47, 115.64, 126.27, 127.92, 129.14, 130.28, 132.88, 134.36, 137.24, 143.08, 148.45, 148.61, 161.13, 163.86, 164.25; MS (ESI m/z): $503.08[\mathrm{M}+\mathrm{H}]^{+}$.

3. 2-(5-(4-chlorophenyl)-3-(thiophen-2-yl)-4,5-dihydro-1H-pyrazol-1-yl)-4'-methyl -2'-phenyl-4,5'-bithiazole (4c)

Yellow solid, yield: (440 mg, 85\%); mp. 256 -258 ${ }^{\circ} \mathrm{C}$; IR (KBr, ख max cm$\left.^{-1}\right)$ : 1610 (C=N), 569 (C-Cl); ${ }^{1} \mathrm{H} \mathrm{NMR}$ (DMSO-d ; $\delta$ ppm): 2.46 (s, 3H, - $\mathrm{CH}_{3}$ ), 3.38 - 3.42 (dd, J = 14 Hz, 5.2 Hz, 1H, Pyrazole-CH2), $4.04-4.10$ (dd, $J=14.4 \mathrm{~Hz}, 9.6 \mathrm{~Hz}, 1 \mathrm{H}$, Pyrazole-CH ), $5.64-5.68$ (dd, J = 9.6 Hz, 5.2 Hz, 1H, Pyrazole-CH), 7.09 (s, 1H, Ar-H), 7.17 - 7.19 (m, 1H, Ar-H), 7.42 - 7.46 (m, 4H, Ar-H), 7.48 - 7.50 (m, 4H, Ar-H), 7.76 (d, J = 4.8 $\mathrm{Hz}, 1 \mathrm{H}, \mathrm{Ar}-\mathrm{H}), 7.89$ (d, $J=6.8 \mathrm{~Hz}, 2 \mathrm{H}, \mathrm{Ar}-\mathrm{H}) ;{ }^{13} \mathrm{C}$ NMR (DMSO-d ${ }_{6}+\mathrm{CDCl}_{3} ; \delta$ ppm): 22.83, 48.34, 61.10, $69.98,110.98,115.29,116.37,123.60,130.73,132.41,133.11,133.69,134.49,138.69,139.46,148.39$, 153.69, 169.25; MS (ESI m/z): $519.05[\mathrm{M}+\mathrm{H}]^{+}$.

4. 2-(5-(4-bromophenyl)-3-(thiophen-2-yl)-4,5-dihydro-1H-pyrazol-1-yl)-4'-methyl -2'-phenyl-4,5'-bithiazole (4d)

Orange solid, yield: (495 mg, 88\%); mp. 296 - $298{ }^{\circ} \mathrm{C}$; IR (KBr, 福ax cm$^{-1}$ ): 1615 (C=N), 598 (C-Br); ${ }^{1} \mathrm{H} \mathrm{NMR}$ (DMSO-d $;$; $\delta$ ppm): 2.51 (s, 3H, - $\mathrm{CH}_{3}$ ), 3.33 - 3.41 (dd, J = 23.6 Hz, 10 Hz, 1H, Pyrazole- $\mathrm{CH}_{2}$ ), $3.91-4.01$ (dd, J = 23.2 Hz, 15.6 Hz, 1H, Pyrazole- $\mathrm{CH}_{2}$ ), $5.50-5.57$ (dd, J = 16 Hz, 10 Hz, 1H, Pyrazole-CH), 6.68 (s, 1H, Ar-H), 7.09 (t, J = 6.8 Hz, 1H, Ar-H), 7.25 (t, J = 10 Hz, 2H, Ar-H), 7.36 (d, J = 10.4 Hz, $1 \mathrm{H}, \mathrm{Ar}-\mathrm{H}), 7.41$ $7.47(\mathrm{~m}, 5 \mathrm{H}, \mathrm{Ar}-\mathrm{H}), 7.63$ (s, 1H, Ar-H) 7.92 (d, J = $10.4 \mathrm{~Hz}, 2 \mathrm{H}, \mathrm{Ar}-\mathrm{H}) ;{ }^{13} \mathrm{C} \mathrm{NMR}$ (DMSO- $\boldsymbol{d}_{6}+\mathrm{CDCl}_{3} ; \delta$ ppm):22.01, 49.02, 60.71, 69.50, 110.08, 116.27, 123.69, 130.88, 132.51, 133.21, 133.69, 134.59, 138.26, 139.37, 148.27, 153.02, 169.19; MS (ESI m/z): $565.00[\mathrm{M}+2 \mathrm{H}]^{+}$.

5. 2-(5-(4-methoxyphenyl)-3-(thiophen-2-yl)-4,5-dihydro-1H-pyrazol-1-yl)-4'-methyl-2'-phenyl-4,5'bithiazole (4e)

Orange solid, yield:( 406 mg, 79\%); mp. 224-226 ${ }^{\circ} \mathrm{C}$; IR (KBr, $\left.\mathbb{X}_{\max } \mathrm{cm}^{-1}\right): 1612(\mathrm{C}=\mathrm{N}), 1265\left(-\mathrm{OCH}_{3}\right) ;{ }^{1} \mathrm{H}$ NMR (DMSO-d $;$; $\delta$ ppm): 2.52 (s, 3H, $-\mathrm{CH}_{3}$ ), 3.32 - 3.38 (dd, J = 17.6 Hz, 6.8 Hz, 1H, Pyrazole-H), 3.77 (s, 3H, $-\mathrm{OCH}_{3}$ ), $3.90-3.98$ (dd, J = 17.2 Hz, 11.6 Hz, 1H, Pyrazole-H), $5.56-5.61$ (dd, J = 11.6 Hz, 6.4 Hz, 1H, 
Pyrazole-H), 6.67 (s, 1H, Ar-H), 6.88 (d, J = 8.4 Hz, 2H, Ar-H), 7.10 (d, J = 4 Hz, 1H, Ar-H), 7.34 (d, J = 8.4 Hz, $2 \mathrm{H}, \mathrm{Ar}-\mathrm{H}), 7.42$ - 7.47 (m, 4H, Ar-H), 7.58 (s, $1 \mathrm{H}, \mathrm{Ar}-\mathrm{H}), 7.89$ (d, J = $7.6 \mathrm{~Hz}, 2 \mathrm{H}, \mathrm{Ar}-\mathrm{H}) ;{ }^{13} \mathrm{C}$ NMR (DMSO-d + $\mathrm{CDCl}_{3}$; $\delta$ ppm): 22.12, 60.03, 69.03, 110.04, 118.81, 130.87, 132.59, 132.88, 133.31, 134.61, 138.05, 139.37, 148.25, 153.16, 153.94, 163.93, 168.98; MS (ESI m/z): $515.10[\mathrm{M}+\mathrm{H}]^{+}$.

6. 2-(5-(3,4-dimethoxyphenyl)-3-(thiophen-2-yl)-4,5-dihydro-1H-pyrazol-1-yl)-4'-methyl-2'-phenyl-4,5'bithiazole (4f)

Yellow solid, yield: (408 mg, 75\%); mp. $232-234^{\circ} \mathrm{C}$; IR (KBr, $\left.\mathbb{M}_{\max } \mathbf{c m}^{-1}\right): 1625(\mathrm{C}=\mathrm{N}), 1256\left(-\mathrm{OCH}_{3}\right) ;{ }^{1} \mathrm{H}$ NMR (DMSO-d $;$; $\delta$ ppm): 2.53 (s, 3H, $-\mathrm{CH}_{3}$ ), 3.33 - 3.41 (dd, J = 23.6 Hz, 9.2 Hz, 1H, Pyrazole-H), 3.85 (s, $\left.3 \mathrm{H},-\mathrm{OCH}_{3}\right), 3.89\left(\mathrm{~s}, 3 \mathrm{H},-\mathrm{OCH}_{3}\right), 3.95-3.99(\mathrm{dd}, \mathrm{J}=23.6 \mathrm{~Hz}, 16.8 \mathrm{~Hz}, 1 \mathrm{H}$, Pyrazole-H), $5.53-5.59$ (dd, J = 16 Hz, 9.6 Hz, 1H, Pyrazole-H), 6.67 (s, 1H, Ar-H), 6.86 (d, J=12 Hz, 1H, Ar-H), 6.96 (s, 2H, Ar-H), 7.10 (d, J $=6.4 \mathrm{~Hz}, 1 \mathrm{H}, \mathrm{Ar}-\mathrm{H}), 7.25$ (s, 1H, Ar-H), $7.41-7.45$ (m, 4H, Ar-H), 7.88 (d, J = 9.2 Hz, 2H, Ar-H); ${ }^{13} \mathrm{C} \mathrm{NMR}$ (DMSO-d ${ }_{6}+$ CDCl$_{3} ; \delta$ ppm): 22.02, 60.71, 69.50, 110.08, 116.27, 123.69, 130.88, 132.51, 133.21, 133.69, 134.59, 153.02, 169.36; MS (ESI m/z): $544.90[\mathrm{M}+\mathrm{H}]^{+}$.

7. 4'-methyl-2'-phenyl-2-(3-(thiophen-2-yl)-5-(3,4,5-trimethoxyphenyl)-4,5-dihydro -1H-pyrazol-1-yl)-4,5'bithiazole (4g)

Yellow solid, yield: (510 mg, 89\%); mp. 250 - 252 ${ }^{\circ}$ C; IR (KBr, $\left.\mathbb{\operatorname { m a x }}_{\max } \mathrm{Cm}^{-1}\right): 1612(\mathrm{C}=\mathrm{N}), 1260\left(-\mathrm{OCH}_{3}\right) ;{ }^{1} \mathrm{H}$ NMR (DMSO-d $;$; $\delta$ ppm): 2.55 (s, 3H, $-\mathrm{CH}_{3}$ ), 3.36 - 3.44 (dd, J = 23.6 Hz,10 Hz, 1H, Pyrazole-CH $), 3.78$ (s, $\left.3 \mathrm{H},-\mathrm{OCH}_{3}\right), 3.86\left(\mathrm{~s}, 3 \mathrm{H},-\mathrm{OCH}_{3}\right), 3.92-4.02$ (dd, J = 23.2 Hz, $15.6 \mathrm{~Hz}, 1 \mathrm{H}$, Pyrazole- $\left.\mathrm{CH}_{2}\right), 5.47-5.54$ (dd, J $=15.6 \mathrm{~Hz}, 10.4 \mathrm{~Hz}, 1 \mathrm{H}$, Pyrazole-CH), 6.65 (s, 2H, Ar-H), 6.77 (s, 1H, Ar-H), 7.11 (t, J = 6.8 Hz, 1H, Ar-H), 7.28 $(\mathrm{d}, \mathrm{J}=3.6 \mathrm{~Hz}, 1 \mathrm{H}, \mathrm{Ar}-\mathrm{H}), 7.44-7.49$ (m, 3H, Ar-H), 7.60 (s, 1H, Ar-H), $7.91(\mathrm{~m}, 1 \mathrm{H}, \mathrm{Ar}-\mathrm{H}){ }^{13} \mathrm{C}$ NMR (DMSO-d + $\mathrm{CDCl}_{3}$; $\delta$ ppm): 21.74, 60.94, 65.28, 70.26, 108.11, 110.79, 130.91, 132.67, 133.88, 135.05, 137.63, 139.17, 141.82, 142.15, 147.76, 153.59, 158.11, 168.43, 169.36\% ; MS (ESI m/z): 575.12 [M+H] ${ }^{+}$

8. 2-(3-(furan-2-yl)-5-(4-methoxyphenyl)-4,5-dihydro-1H-pyrazol-1-yl)-4'-methyl-2'-phenyl-4,5'-bithiazole (4h)

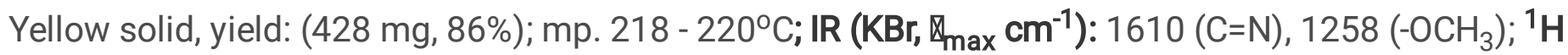
NMR (DMSO-d $;$; ppm): $2.63\left(\mathrm{~s}, 3 \mathrm{H},-\mathrm{CH}_{3}\right), 3.58$ - 3.66 (dd, J = 23.2 Hz, 9.2 Hz, 1H, Pyrazole-CH $), 3.71-$ 3.81 (dd, $J=22.8 \mathrm{~Hz}, 15.6 \mathrm{~Hz}, 1 \mathrm{H}$, Pyrazole $\mathrm{CH}_{2}$ ), $3.86\left(\mathrm{~s}, 3 \mathrm{H},-\mathrm{OCH}_{3}\right), 5.67-5.73$ (dd, J = 15.6 Hz, 9.2 Hz, 1H, Pyrazole-CH), 6.36 - 6.38 (m, 1H, Ar-H), 6.55 (d, J = 4 Hz, 1H, Ar-H), 6.70 (s, 1H, Ar-H), 6.96 (d, J= 11.6 $\mathrm{Hz}, 2 \mathrm{H}, \mathrm{Ar}-\mathrm{H}), 7.37$ - 7.52 (m, 4H, Ar-H), 7.72 (d, J = 11.6 Hz, 2H, Ar-H), 7.92 (d, J = 9.6 Hz, 2H, Ar-H); ${ }^{13} \mathrm{C}$ NMR (DMSO- $d_{6}+\mathrm{CDCl}_{3} ; \delta$ ppm): 22.17, 47.75, 60.19, 62.48, 110.00, 113.86, 115.40, 118.99, 128.44, 130.90, 132.86, 133.74, 134.60, 138.35, 147.00, 148.21, 153.88, 156.37, 157.41, 165.89, 168.59, 169.51; MS (ESI m/z): $499.15[\mathrm{M}+\mathrm{H}]^{+}$. 
9. 2-(5-(3,4-dimethoxyphenyl)-3-(pyridin-2-yl)-4,5-dihydro-1H-pyrazol-1-yl)-4'-methyl-2'-phenyl-4,5'bithiazole (4i)

Yellow solid, yield: (485 mg, 90\%); mp. 262 - 264 ${ }^{\circ} \mathrm{C}$; IR (KBr, $\left.\mathbb{X}_{\max } \mathrm{cm}^{-1}\right): 1622(\mathrm{C}=\mathrm{N}), 1248\left(-\mathrm{OCH}_{3}\right) ;{ }^{1} \mathrm{H}$

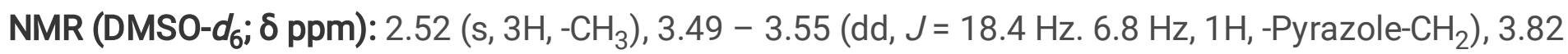
$\left(\mathrm{s}, 3 \mathrm{H},-\mathrm{OCH}_{3}\right), 3.87\left(\mathrm{~s}, 3 \mathrm{H},-\mathrm{OCH}_{3}\right), 4.00-4.04\left(\mathrm{dd}, J=12 \mathrm{~Hz}, 4 \mathrm{~Hz}, 1 \mathrm{H}\right.$, Pyrazole $\left.-\mathrm{CH}_{2}\right), 5.56-5.61$ (dd, $J$ $=12.4 \mathrm{~Hz}, 7.2 \mathrm{~Hz}, 1 \mathrm{H}$, Pyrazole-CH), $6.71(\mathrm{~s}, 1 \mathrm{H}, \mathrm{Ar}-\mathrm{H}), 6.84(\mathrm{~d}, J=8 \mathrm{~Hz}, 1 \mathrm{H}, \mathrm{Ar}-\mathrm{H}), 6.96(\mathrm{~d}, J=8.4 \mathrm{~Hz}, 1 \mathrm{H}$, Ar-H), $7.29-7.32(\mathrm{~m}, 1 \mathrm{H}, \mathrm{Ar}-\mathrm{H}), 7.33-7.40(\mathrm{~m}, 3 \mathrm{H}, \mathrm{Ar}-\mathrm{H}), 7.59(\mathrm{~s}, 1 \mathrm{H}, \mathrm{Ar}-\mathrm{H}), 7.91(\mathrm{t}, J=8 \mathrm{~Hz}, 1 \mathrm{H}, \mathrm{Ar}-\mathrm{H})$, $7.87(\mathrm{~d}, J=8.6 \mathrm{~Hz}, 2 \mathrm{H}, \mathrm{Ar}-\mathrm{H}), 8.13(\mathrm{~d}, J=8 \mathrm{~Hz}, 1 \mathrm{H}, \mathrm{Ar}-\mathrm{H}), 8.59$ (d, $J=5.6 \mathrm{~Hz}, 1 \mathrm{H}, \mathrm{Ar}-\mathrm{H}){ }^{13} \mathrm{C}$ NMR (DMSO- $d_{6}$ + $\mathrm{CDCl}_{3} ; \delta$ ppm): 22.11, 48.00, 60.71, 69.63, 110.36, 115.56, 116.44, 123.79, 126.01, 128.88, 130.82, 133.74, 134.62, 138.83, 141.19, 148.32, 154.17, 158.56, 168.47, 169.04; MS (ESI m/z): $540.15[\mathrm{M}+\mathrm{H}]^{+}$.

10. 2-(3-(benzofuran-2-yl)-5-(4-chlorophenyl)-4,5-dihydro-1H-pyrazol-1-yl)-4'-methyl-2'-phenyl-4,5'bithiazole (4j)

Yellow solid, yield: (508 mg, 92\%); mp. $236-238^{\circ} \mathrm{C}$; IR (KBr, $\left.\mathbb{Z}_{\max } \mathrm{cm}^{-1}\right): 1612$ (C=N), 725 (C-Cl); ${ }^{1} \mathrm{H}$ NMR

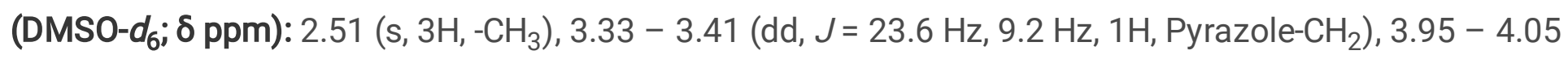
(dd, $J=23.2 \mathrm{~Hz}, 16 \mathrm{~Hz}, 1 \mathrm{H}$, Pyrazole-CH), $5.64-5.70$ (dd, $J=16.4 \mathrm{~Hz}, 9.2 \mathrm{~Hz}, 1 \mathrm{H}$, Pyrazole-CH $)_{2}, 6.72$ (s, 1H, -Ar-H), 7.09 (s, 1H, Ar-H), $7.35-7.45$ (m, 9H, Ar-H), $7.56-7.64$ (m, 2H, Ar-H), 7.90 (d, J = 10.4 Hz, 2H, Ar-H); ${ }^{13} \mathrm{C}$ NMR (DMSO- $d_{6} ; \delta$ ppm): 17.42, 43.11, 63.78, 107.10, 110.50,111.82, 122.34, 124.36, 126.42, 128.08, 129.12, 132.89, 140.31, 143.05, 144.96, 148.11, 155.54, 163.96; MS (ESI m/z): $554.10[\mathrm{M}+\mathrm{H}]^{+}$.

11. 2-(3-(benzofuran-2-yl)-5-(4-methoxyphenyl)-4,5-dihydro-1H-pyrazol-1-yl)-4'-methyl-2'-phenyl-4,5'bithiazole (4k)

Yellow solid, yield: (499 mg, 91\%); mp. $287-289^{\circ} \mathrm{C}$; IR (KBr, $\left.\mathbb{X}_{\max } \mathrm{cm}^{-1}\right): 1630(\mathrm{C}=\mathrm{N}), 1245\left(\mathrm{C}-\mathrm{OCH}_{3}\right) ;{ }^{1} \mathbf{H}$

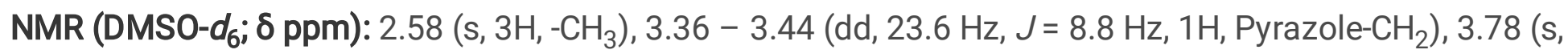
$3 \mathrm{H},-\mathrm{OCH}_{3}$ ), $3.93-4.02$ (dd, $J=23.2 \mathrm{~Hz}, 16 \mathrm{~Hz}, 1 \mathrm{H}$, Pyrazole-CH), $5.62-5.68$ (dd, $J=16 \mathrm{~Hz}, 8.8 \mathrm{~Hz}, 1 \mathrm{H}$, Pyrazole-CH), 6.73 (s, 1H, Ar-H), 6.89 (d, J=11.6 Hz, 2H, Ar-H), $7.08(\mathrm{~s}, 1 \mathrm{H}, \mathrm{Ar}-\mathrm{H}), 7.28(\mathrm{t}, J=10.4 \mathrm{~Hz}, 1 \mathrm{H}$, Ar-H), 7.34 - 7.37 (m, 3H, Ar-H), $7.44-7.47$ (m, 3H, Ar-H), $7.56-7.64$ (m, 2H, Ar-H), 7.94 (s, 2H, Ar-H); ${ }^{13} \mathrm{C}$ NMR (DMSO- $d_{6}+\mathrm{CDCl}_{3} ; \delta$ ppm): 22.01, 47.75, 60.04, 68.81, 110.36, 113.34, 116.32, 118.84, 126.47, $128.34,130.95,132.87,133.75,134.71,137.74,148.59,160.23,164.00,168.67 ;$ MS (ESI m/z): 549.10 $[\mathrm{M}+\mathrm{H}]^{+}$.

\section{Results And Discussion}

\section{Chemistry:}

The required starting material chalcones (3a-k) for the preparation of target compounds (4a-k), were obtained by reacting 2-Acetylfuran / 2-Acetylthiphene / 2-Acetylbenzofuran 
with various substituted aldehydes in the presence of $\mathrm{NaOH}$ in ethanol at $0^{\circ} \mathrm{C}$ to room temperature as per the literature procedure $[50,51]$. The title compounds dihydro pyrazolyl bithiazole derivatives $(4 a-k)$, were synthesized via multicomponent reaction approach by the condensation of an equimolar mixture of 2bromo-1-(4-methyl-2-phenyl thiazol-5-yl)ethan-1-one (1), thiosemicarbazide (2) and substituted chalcones ( $3 \mathrm{a}-\mathrm{k})$ in the presence of sodium hydroxide in ethanol under reflux with good yields (Table 1) as outlined in Scheme 1.

To know the feasibility of the reaction, initially we have carried out the reaction of 2-bromo-1-(4-methyl-2phenyl thiazol-5-yl)ethan-1-one (1), thiosemicarbazide (2) and substituted chalcone (3a) in presence of absolute ethanol as a solvent for about $12-16 \mathrm{~h}$ reaction time at $70^{\circ} \mathrm{C}$. The yield of the reaction was very poor and the reaction time was very high. In order to improve the yield of the reactions and to reduce the reaction time we tried the reaction by using $\mathrm{Na}_{2} \mathrm{CO}_{3}, \mathrm{~K}_{2} \mathrm{CO}_{3}, \mathrm{NaOH}$ and $\mathrm{KOH}$ with different molar ratios (1eq, 1.5 eq, 2 eq and 3 eq). Among these variations, 1.5 eq. $\mathrm{NaOH}$ at $70^{\circ} \mathrm{C}$ gave the product with good yield within shorter reaction time (4- $5 \mathrm{~h}$ ). Here we reported reaction optimization conditions in Table 2.

A plausible reaction mechanism for target molecules 4(a-k) was shown in Scheme 2. Initially thiosemicarbazide (2) reacts with 2-bromo-1-(4-methyl-2-phenylthiazol-5-yl)ethan-1-one (1) and forms Hantzsch thiazole by the elimination of $\mathrm{HBr}$ and $\mathrm{H}_{2} \mathrm{O}$. Subsequent condensation of a-hydrazinothiazole with $a, \beta$ unsaturated ketones could result the formation of dihydropyrazole ring. Overall the three component domino transformation leads to three $\mathrm{C}-\mathrm{N}$ and one $\mathrm{C}-\mathrm{S}$ bonds. In this three component reaction two heterocyclic systems, thiazole and dihydropyrazoles were successfully synthesized.

The structures of the newly synthesized compounds were confirmed by IR, ${ }^{1} \mathrm{H}$ NMR, ${ }^{13} \mathrm{C}$ NMR and Mass spectral data and described here in detail for a representative compound $4 \mathrm{~b} .{ }^{1} \mathrm{H}$ NMR spectrum of compound $4 \mathrm{~b}$, the three protons present at fourth and fifth position of pyrazoline ring exhibited characteristic ABX splitting pattern and displayed double doublets (dd). The chemical shift values for $\mathrm{Ha}$ proton appeared at $\delta 3.29-3.34 \mathrm{ppm}(\mathrm{dd}, J=12.8 \mathrm{~Hz}, 6 \mathrm{~Hz}, 1 \mathrm{H})$, for $\mathrm{Hb}$ proton appeared at $\delta$ 3.97- 4.04 ppm (dd, $J=16.8 \mathrm{~Hz}, 4.8 \mathrm{~Hz}, 1 \mathrm{H})$, for $\mathrm{Hx}$ proton chemical shift value appeared at $\delta 5.61-5.66 \mathrm{ppm}(\mathrm{dd}, J$ $=11.2 \mathrm{~Hz}, 5.6 \mathrm{~Hz}, 1 \mathrm{H}$ ) and the remaining aromatic protons appeared in the range of $\delta 7.07-7.90 \mathrm{ppm}$ respectively. ${ }^{13} \mathrm{C}$ NMR has been recorded for the compounds $4 a-k$ and the characteristic peaks of dihydropyrazole C3, C4 and C5 are exhibited in the range of $\delta 163.5,44.3$ and $64.7 \mathrm{ppm}$ respectively. The ESI-Mass spectra were recorded for the compounds $4 \mathrm{a}-\mathrm{k}$ and all the compounds exhibited $(\mathrm{M}+\mathrm{H})^{+}$as base peak. All the spectral analysis data of the final compounds clearly indicates the formation of target compounds.

Table 1. Synthesis of dihydro pyrazolyl bithiazole derivatives 4a-k: 


\begin{tabular}{llllll} 
S.No. & Product & $\mathrm{R}$ & $\mathrm{R}^{1}$ & $\mathrm{R}^{2}$ & $\mathrm{R}^{3}$ \\
\hline 1 & $4 \mathrm{a}$ & thiophen-2-yl & $\mathrm{H}$ & $\mathrm{H}$ & $\mathrm{H}$ \\
\hline 2 & $4 \mathrm{~b}$ & thiophen-2-yl & $\mathrm{H}$ & $\mathrm{F}$ & $\mathrm{H}$ \\
\hline 3 & $4 \mathrm{c}$ & thiophen-2-yl & $\mathrm{H}$ & $\mathrm{Cl}$ & $\mathrm{H}$ \\
\hline 4 & $4 \mathrm{~d}$ & thiophen-2-yl & $\mathrm{H}$ & $\mathrm{Br}$ & $\mathrm{H}$ \\
\hline 5 & $4 \mathrm{e}$ & thiophen-2-yl & $\mathrm{H}$ & $-\mathrm{OCH}_{3}$ & $\mathrm{H}$ \\
\hline 6 & $4 \mathrm{f}$ & thiophen-2-yl & $-\mathrm{OCH}_{3}$ & $-\mathrm{OCH}_{3}$ & $\mathrm{H}$ \\
\hline 7 & $4 \mathrm{~g}$ & thiophen-2-yl & $-\mathrm{OCH}_{3}$ & $-\mathrm{OCH}_{3}$ & $-\mathrm{OCH}$ \\
\hline 8 & $4 \mathrm{~h}$ & furan-2-yl & $\mathrm{H}$ & $-\mathrm{OCH}_{3}$ & $\mathrm{H}$ \\
\hline 9 & $4 \mathrm{i}$ & pyridin-2-yl & $-\mathrm{OCH}_{3}$ & $-\mathrm{OCH}_{3}$ & $\mathrm{H}$ \\
\hline 10 & $4 \mathrm{j}$ & benzofuran-2-yl & $\mathrm{H}$ & $\mathrm{Cl}^{2}$ & $\mathrm{H}$ \\
\hline 11 & $4 \mathrm{k}$ & benzofuran-2-yl & $\mathrm{H}$ & $-\mathrm{OCH}_{3}$ & $\mathrm{H}$
\end{tabular}

Table 2. Reaction optimization conditions for the synthesis of dihydro pyrazolyl bithiazole derivatives:

\section{Anticancer activity application of 2-(5-(Aryl)-3-heteryl-4,5-dihydro-1H-pyrazol-1-yl)-4'-methyl-2'-phenyl-4,5'- bithiazole derivatives (4a-k):}

The newly synthesized 2-(5-(Aryl)-3-heteryl-4,5-dihydro-1H-pyrazol-1-yl)-4'-methyl-2'-phenyl-4,5'-bithiazole derivatives (4a-k) were evaluated for anticancer activity application by using standard in vitro MTT assay [52] with four different human cancer cell lines like Non-small-cell lung cancer cell lines (A549), prostate cancer (DU-145), breast cancer (MCF-7), and Human neuroblastoma (SKNSH). The preliminary investigation of anticancer properties of the novel compounds (4a-K) were done at 50 and $100 \mu \mathrm{M}$ concentrations and percentage cell line growth inhibition was measured. From the preliminary anticancer activity results, majority of the compounds exhibited profound cell growth inhibition. All the newly synthesized compounds were further screened at various concentrations $(1-100 \mu \mathrm{M})$ and IC ${ }_{50}$ values were calculated with respect to standard Doxorubicin, by repeating the experiments in thrice and the average values were shown in Table 3.

Table 3. Anticancer activity evaluation ( $\mathrm{IC}_{50}$ values) of novel 2-(5-(Aryl)-3-heteryl-4,5-dihydro-1H-pyrazol-1yl)-4'-methyl-2'-phenyl-4,5'-bithiazole derivatives (4a-k): 


\begin{tabular}{|c|c|c|c|c|c|}
\hline Entry & Solvent & Base & Temperature $\left({ }^{\circ} \mathrm{C}\right)$ & Time (h) & Yield (\%) \\
\hline \multirow{5}{*}{1} & \multirow{5}{*}{ Methanol } & - & \multirow{5}{*}{ reflux } & 16 & 20 \\
\hline & & $\mathrm{Na}_{2} \mathrm{CO}_{3}$ & & 8 & 45 \\
\hline & & $\mathrm{K}_{2} \mathrm{CO}_{3}$ & & 8 & 50 \\
\hline & & $\mathrm{NaOH}$ & & 6 & 65 \\
\hline & & $\mathrm{KOH}$ & & 6 & 60 \\
\hline \multirow{5}{*}{2} & \multirow{5}{*}{ Ethanol } & - & \multirow{5}{*}{ reflux } & 16 & 45 \\
\hline & & $\mathrm{Na}_{2} \mathrm{CO}_{3}$ & & 12 & 50 \\
\hline & & $\mathrm{K}_{2} \mathrm{CO}_{3}$ & & 10 & 55 \\
\hline & & $\mathrm{NaOH}$ & & 4 & 85 \\
\hline & & $\mathrm{KOH}$ & & 6 & 80 \\
\hline 3 & Acetonitrile & - & reflux & 16 & 25 \\
\hline 4 & Dimethylformamide & - & 120 & 16 & 40 \\
\hline 5 & Dimethylsulphoxide & - & 120 & 16 & 35 \\
\hline 6 & Acetic acid & - & 110 & 16 & 50 \\
\hline
\end{tabular}




\section{$\mathrm{IC}_{50}(\mu \mathrm{M})$}

\begin{tabular}{llllll} 
S.No. & Compound & A549 & DU145 & MCF7 & SKNSH \\
\hline 1 & $4 \mathrm{a}$ & $13.88 \pm 0.21$ & $\mathbf{9 . 4 1} \pm \mathbf{0 . 3 2}$ & $\mathbf{4 . 6 2} \pm \mathbf{0 . 3 4}$ & $\mathbf{8 . 1 7} \pm \mathbf{0 . 3 1}$ \\
\hline 2 & $4 \mathrm{~b}$ & $19.68 \pm 0.11$ & $11.31 \pm 0.12$ & $14.92 \pm 0.14$ & $17.57 \pm 0.11$ \\
\hline 3 & $4 \mathrm{c}$ & $47.71 \pm 0.28$ & $\mathbf{9 . 2 1} \pm \mathbf{0 . 1 4}$ & $14.43 \pm 0.62$ & $19.42 \pm 0.34$ \\
\hline 4 & $4 \mathrm{~d}$ & $75.26 \pm 0.38$ & $16.43 \pm 0.16$ & $\mathbf{7 . 1 7} \pm \mathbf{0 . 3 3}$ & $18.23 \pm 0.34$ \\
\hline 5 & $4 \mathrm{e}$ & $20.86 \pm 0.34$ & $14.98 \pm 0.21$ & $11.64 \pm 0.24$ & $13.2 \pm 0.42$ \\
\hline 6 & $4 \mathrm{f}$ & $20.54 \pm 0.42$ & $20.42 \pm 0.72$ & $\mathbf{3 . 4 7} \pm \mathbf{0 . 2 8}$ & $\mathbf{9 . 1 4} \pm \mathbf{0 . 1 2}$ \\
\hline 7 & $4 \mathrm{~g}$ & $24.93 \pm 0.19$ & $12.83 \pm 0.37$ & $\mathbf{1 . 3 6} \pm \mathbf{0 . 7 4}$ & $11.44 \pm 0.73$ \\
\hline 8 & $4 \mathrm{~h}$ & $17.92 \pm 0.32$ & $22.63 \pm 0.18$ & $\mathbf{3 . 7 6} \pm \mathbf{0 . 3 5}$ & $16.67 \pm 0.12$ \\
\hline 9 & $4 \mathrm{i}$ & $21.64 \pm 0.36$ & $\mathbf{8 . 3 6} \pm \mathbf{0 . 3 2}$ & $15.65 \pm 0.28$ & $\mathbf{5 . 3 6} \pm \mathbf{0 . 2 3}$ \\
\hline 10 & $4 \mathrm{j}$ & $24.27 \pm 0.16$ & $15.51 \pm 0.34$ & $\mathbf{4 . 1 4} \pm \mathbf{0 . 2 6}$ & $12.52 \pm 0.24$ \\
\hline 11 & $4 \mathrm{k}$ & $25.84 \pm 0.17$ & $20.38 \pm 0.24$ & $13.51 \pm 0.15$ & $18.6 \pm 0.48$ \\
\hline 12 & Doxorubicin & $1.37 \pm 0.11$ & $3.72 \pm 0.14$ & $1.29 \pm 0.16$ & $2.18 \pm 0.32$ \\
\hline
\end{tabular}

From the anticancer activity evaluation results, all the compounds exhibited good to moderate anticancer activity against the four screened human cancer cell lines. Compound $4 \mathrm{~g}$ exhibited excellent activity against MCF-7 cancer cell line with $\mathrm{IC}_{50}$ value of $1.36 \pm 0.74 \mu \mathrm{M}$. Among the tested eleven compounds, compound 4 a exhibited promising activity against DU-145, MCF-7and SKNSH cancer cell lines with $\mathrm{IC}_{50}$ values of $9.41 \pm 0.32,4.62 \pm 0.34$ and $8.17 \pm 0.31 \mu \mathrm{M}$ concentrations. Compounds $4 \mathrm{c}$ and $4 \mathrm{i}$ were shown good activity against DU-145 cell line with $\mathrm{IC}_{50}$ values of $9.21 \pm 0.14$ and $8.36 \pm 0.32 \mu \mathrm{M}$ concentrations. Majority of the compounds like $4 \mathrm{~d}, 4 \mathrm{f}, 4 \mathrm{~h}$ and $4 \mathrm{j}$ have shown promising anticancer property against MCF7 cell line with $\mathrm{IC}_{50}$ values of $7.17 \pm 0.33,3.47 \pm 0.28,3.76 \pm 0.35$ and $4.14 \pm 0.26 \mu \mathrm{M}$ concentrations. Whereas the compound $4 \mathrm{f}$ and $4 \mathrm{i}$ exhibited good anticancer property against SKNSH cancer cell line with $\mathrm{IC}_{50}$ values of $9.14 \pm 0.12$ and $5.36 \pm 0.23 \mu \mathrm{M}$ concentrations respectively.

\section{In silico studies of the2-(5-(Aryl)-3-heteryl-4,5-dihydro-1H-pyrazol-1-yl)-4'-methyl-2'-phenyl-4,5'-bithiazole derivatives:}

The in vitro results suggest that breast cancer cell line is more effected by the novel 2-(5-(Aryl)-3-heteryl4,5-dihydro-1 H-pyrazol-1-yl)-4'-methyl-2'-phenyl-4,5'-bithiazole derivatives (4a-k) than the other cell lines. The cytotoxicity result has been validated by docking simulation study using AutoDock Vina (ADT Vina) [53]. Docking results elucidate the importance of different types of interactions to inhibit the function of probable target for breast cancer lines, Human Estrogen Receptor Alpha protein (3ert.pdb) [54]. In the present study only three ligands, $4 \mathrm{f}, 4 \mathrm{~g}$ and $4 \mathrm{i}$ with the residue Cys 530, Meth 522 and Thr 347 form hydrogen binding respectively. 
The binding affinity of all the novel derivatives has stronger binding energies than that of the bound ligand OHT. The docking energy values of derivatives are given in Table 3 with their active site residues. Among all the ligands, compound $4 \mathrm{j}$ having a good binding affinity compared to co-crystalized bound ligand $\mathrm{OHT}$. The pattern of cytotoxic activity of derivatives is matching more than $75 \%$ of in silico results.

The order of binding affinity of selected ligands against the target is $\mathbf{4 j}>\mathbf{4 a}>\mathbf{4 i}>\mathbf{4 d}>\mathbf{4 h}>\mathbf{4 b}>\mathbf{4 c}>\mathbf{4 g}>$ $\mathbf{4 f}>\mathbf{4 e}>\mathbf{4 k}$ and $\mathrm{OHT}$ with binding energy range -7.8 to $-10.1 \mathrm{k} \mathrm{cal} / \mathrm{mol}$ (Table 4).

Table 4. In silico activity evaluation ligands (4a-k) using ADT Vina against the target Human Estrogen Receptor Alpha protein (3ert.pdb):

\section{S. Name of Docking Active site residues of receptor (3ert.pdb) \\ No. Ligands Energy \\ (kcal/mol)}

$\begin{array}{lll}\text { 1. } & \text { Leu 346, Thr 347, Ala 350, Asp 351, Trp 383, Leu 384, Met 522, Leu } \\ & \text { 525, Tyr 526. Leu } 536\end{array}$

2. $-8.7 \quad$ Met 343, Thr 347, Ala 350, Trp 383, Met 522, Leu 525, Try 526, Cys $4 b$ 530, Lys 531, Val 533, Pro 535

\begin{tabular}{|c|c|c|c|}
\hline 3. & $4 c$ & -8.4 & $\begin{array}{l}\text { Met 343, Thr 347, Ala 350, Trp 383, Asn 519, Met 522, Glu 523, Leu } \\
\text { 525, Try } 526\end{array}$ \\
\hline 4. & $4 d$ & -8.8 & $\begin{array}{l}\text { Leu 346, Thr 347, Ala 350, Asp 351, Trp 383, Met 522, Leu 525, Tyr } \\
\text { 526, Leu 536, Leu 539 }\end{array}$ \\
\hline 5. & $4 e$ & -7.8 & $\begin{array}{l}\text { Ala 350, Asp 351, Leu 354, Glu 380, Trp 383, Leu 525, Tyr 526, Lys } \\
\text { 529, Cys } 530\end{array}$ \\
\hline 6. & $4 f$ & -7.9 & $\begin{array}{l}\text { Met 522, Glu 523, Tyr 526, Leu 529, Cys 530, Val 533, Val 534, Leu } \\
\text { 535, Leu } 536\end{array}$ \\
\hline 7. & $4 \mathrm{~g}$ & -8.2 & $\begin{array}{l}\text { Ala 350, Asp 351, Leu 354, Glu 380, Trp 383, Met 522, Leu 525, Tyr } \\
\text { 526, Cys 530, Val 533, Pro 535, Leu } 536\end{array}$ \\
\hline 8. & $4 \mathrm{~h}$ & -8.8 & $\begin{array}{l}\text { Leu 346, Thr 347, Ala 350, Asp 351, Trp 383, Leu 525, Val 533, Tyr } \\
\text { 534, Leu 536, Leu 539 }\end{array}$ \\
\hline 9. & $4 i$ & -8.9 & $\begin{array}{l}\text { Leu 346, Thr 347, Ala 350, Asp 351, Trp 383, Leu 525, Tyr 526, Met } \\
\text { 533, Val 534, Leu 536, Met } 543\end{array}$ \\
\hline 10. & $4 j$ & -10.1 & $\begin{array}{l}\text { Leu 346, Thr 347, Ala 350, Asp 351, Trp 383, Leu 384, Leu 525, Met } \\
\text { 528, Lys 529, Met 533, Pro 535, Leu 536, Leu } 539\end{array}$ \\
\hline 11. & $4 \mathrm{k}$ & -7.8 & $\begin{array}{l}\text { Leu 346, Thr 347, Ala 350, Asp 351, Trp 383, Leu 384, Met 528, Lys } \\
\text { 529, Met 533, Pro 535, Leu 536, Leu } 539\end{array}$ \\
\hline 12. & $\mathrm{OHT}$ & -7.8 & $\begin{array}{l}\text { Ala 350, Asp 351, Leu 354, Trp 383, Met 522, Tyr } 526 \text { Cys 530, Val } \\
\text { 533, Leu } 536\end{array}$ \\
\hline
\end{tabular}


With the help of comparative analysis of active site residues interactions with the ligands shows that Thr 347, Asp 351, TYR 526, Leu 536, Leu 525, Ala 350, Trp 383 are key residue of catalytic site of Estrogen Receptor Alpha protein (3ert.pdb) and commonly occurs 8, 8, 8, 9, 10, 10 and 11 times respectively. The bound confirmation of docked ligands depicted that all are occupy the same binding cavity and shown in figure 2 with secondary structure of receptor (3ert.pdb). The 2-D \& 3-D representations are shown in figure $3 a-c$ for ligand $4 \mathrm{~g}$.

\section{Conclusion}

In summary, a series of 2-(5-(Aryl)-3-heteryl-4,5-dihydro-1 H-pyrazol-1-yl)-4'-methyl-2'-phenyl-4,5'-bithiazole derivatives (4a-k) were synthesized efficiently via one pot multicomponent reaction approach with good yields and evaluated for their anticancer activity properties. The method applied for the novel targets is simple and highly efficient in shorter reaction time. Most of the newly synthesized compounds were shown promising anticancer activity against breast cancer (MCF-7) cell line.

\section{Declarations}

\section{Acknowledgements:}

The authors thank Department of Chemistry, Osmania University, Hyderabad for laboratory infrastructure under SAP (DRS-I) and DST-FIST programmes.

\section{Conflict of Interest:}

The authors declare no conflict of interest.

\section{References}

1. Kong L, Huang R, He H, Fan Y, Lin J, Yan S (2020) Multi-component solvent-free cascade reaction of 2-cyanoacetamides: regioselective synthesis of pyridin-2-ones bearing quaternary centers. Green Chem 22(1):256-264

2. Victor Snieckus D, Chandra Mohan DFW Multicomponent Mannich Reaction through $\mathrm{C}-\mathrm{H}$ Activation of Azabiaryls.Synfacts. 2020 Jan13;16(01):0020

3. Mega RS, Duong VK, Noble A, Aggarwal VK (2020 Mar) Decarboxylative Conjunctive Cross-coupling of Vinyl Boronic Esters using Metallaphotoredox Catalysis. Angew Chemie Int Ed 9;59((11):43754379

4. Paul B, Maji M, Chakrabarti K, Kundu S (2020) Tandem transformations and multicomponent reactions utilizing alcohols following dehydrogenation strategy. Org Biomol Chem 18(12):21932114

5. Kalinski C, Umkehrer M, Weber L, Kolb J, Burdack C, Ross G On the industrial applications of MCRs: molecular diversity in drug discovery and generic drug synthesis.Mol Divers. 2010 Aug16;14(3):513- 
6. Ruijter E, Orru RVA (2013 Mar) Multicomponent reactions - opportunities for the pharmaceutical industry. Drug Discov Today Technol 10(1):e15-20

7. Capon RJ, Peng C, Dooms C (2008) Trachycladindoles A-G: cytotoxic heterocycles from an Australian marine sponge, Trachycladus laevispirulifer. Org Biomol Chem 6(15):2765-2771

8. Capon RJ, Ratnayake R, Stewart M, Lacey E, Tennant S, Gill JH (2005) Aspergillazines A-E: novel heterocyclic dipeptides from an Australian strain of Aspergillus unilateralis. Org Biomol Chem 3(1):123-129

9. Watson AA, Fleet GWJ, Asano N, Molyneux RJ, Nash RJ (2001 Feb) Polyhydroxylated alkaloids natural occurrence and therapeutic applications. Phytochemistry 56(3):265-295

10. Lamberth C (2013 Oct) Heterocyclic chemistry in crop protection. Pest Manag Sci 69(10):1106-1114

11. Hua X, Liu W, Su Y, Liu X, Liu J, Liu N et al (2020 Jul) Studies on the novel pyridine sulfide containing SDH based heterocyclic amide fungicide. Pest Manag Sci 20;76((7):2368-2378

12. Dong H, Xu S, Wang J, Zhang H, Chen Y, Bi L et al (2020) Synthesis and herbicidal application of turpentine derivative $\mathrm{p}$-menthene type secondary amines as sustainable agrochemicals. New $\mathrm{J}$ Chem 44(20):8280-8288

13. Maffeis V, Moni L, Di Stefano D, Giordani S, Riva R (2020 Feb) Diversity-oriented synthesis of blue emissive nitrogen heterocycles and their conjugation with carbon nano-onions. Front Chem Sci Eng 22;14((1):76-89

14. Klimek-Szczykutowicz, Szopa A, Ekiert H Citrus limon (Lemon) Phenomenon-A Review of the Chemistry, Pharmacological Properties, Applications in the Modern Pharmaceutical, Food, and Cosmetics Industries, and Biotechnological Studies.Plants 2020 Jan17;9(1):119

15. Kreidl M, Rainer M, Jakschitz T, Bonn GK (2020 Mar) Determination of phototoxic furanocoumarins in natural cosmetics using SPE with LC-MS. Anal Chim Acta 1101(xxxx):211-221

16. Wang L-L, Battini N, Bheemanaboina RRY, Zhang S-L, Zhou C-H (2019 Apr) Design and synthesis of aminothiazolyl norfloxacin analogues as potential antimicrobial agents and their biological evaluation. Eur J Med Chem 167:105-123

17. Olawode EO, Tandlich R, Prinsloo E, Isaacs M, Hoppe H, Seldon R et al (2019 Jul) Synthesis and biological evaluation of 2-chloro-3-[(thiazol-2-yl)amino]-1,4-naphthoquinones. Bioorg Med Chem Lett 29(13):1572-1575

18. Gürsoy E, Dincel ED, Naesens L, Ulusoy Güzeldemirci N (2020 Jan) Design and synthesis of novel Imidazo[2,1-b]thiazole derivatives as potent antiviral and antimycobacterial agents. Bioorg Chem 95:103496

19. Singh IP, Gupta S, Kumar S (2020 Jan) Thiazole Compounds as Antiviral Agents: An Update. Med Chem (Los Angeles) 16;16((1):4-23

20. Lamut A, Gjorgjieva M, Naesens L, Liekens S, Lillsunde K-E, Tammela P et al (2020 May) Antiinfluenza virus activity of benzo[d]thiazoles that target heat shock protein 90 . Bioorg Chem 
98(March):103733

21. Karale UB, Krishna VS, Krishna EV, Choudhari AS, Shukla M, Gaikwad VR et al (2019 Sep) Synthesis and biological evaluation of 2,4,5-trisubstituted thiazoles as antituberculosis agents effective against drug-resistant tuberculosis. Eur J Med Chem 178:315-328

22. Mishra I, Mishra R, Mujwar S, Chandra P, Sachan N A retrospect on antimicrobial potential of thiazole scaffold.J Heterocycl Chem. 2020 Jun30;57(6):2304-29

23. Ansari M, Shokrzadeh M, Karima S, Rajaei S, Fallah M, Ghassemi-Barghi N et al (2020 Jan) New thiazole-2(3H)-thiones containing 4-(3,4,5-trimethoxyphenyl) moiety as anticancer agents. Eur $\mathrm{J}$ Med Chem 185:111784

24. Sayed AR, Gomha SM, Taher EA, Muhammad ZA, El-Seedi HR, Gaber HM et al (2020 Apr) One-Pot Synthesis of Novel Thiazoles as Potential Anti-Cancer Agents. Drug Des Devel Ther 14:1363-1375

25. Farghaly TA, Masaret GS, Muhammad ZA, Harras MF (2020 May) Discovery of thiazole-basedchalcones and 4-hetarylthiazoles as potent anticancer agents: Synthesis, docking study and anticancer activity. Bioorg Chem 98:103761

26. de Siqueira LRP, de Moraes Gomes PAT, de Lima Ferreira LP, de Melo Rêgo MJB, Leite ACL (2019 May) Multi-target compounds acting in cancer progression: Focus on thiosemicarbazone, thiazole and thiazolidinone analogues. Eur J Med Chem 170:237-260

27. Jacob PJ, Manju SL (2020 Jul) Identification and development of thiazole leads as COX-2/5-LOX inhibitors through in-vitro and in-vivo biological evaluation for anti-inflammatory activity. Bioorg Chem 100(January):103882

28. Ahmed A, Molvi KI, Patel HM, Ullah R, Bari A (2020 Apr) Synthesis of novel 2, 3, 5-tri-substituted thiazoles with anti-inflammatory and antibacterial effect causing clinical pathogens. $J$ Infect Public Health 13(4):472-479

29. Ghonim AE, Ligresti A, Rabbito A, Mahmoud AM, Di Marzo V, Osman NA et al (2019 Oct) Structureactivity relationships of thiazole and benzothiazole derivatives as selective cannabinoid CB2 agonists with in vivo anti-inflammatory properties. Eur J Med Chem 180:154-170

30. Georgiadis M, Kourbeli V, Papanastasiou IP, Tsotinis A, Taylor MC, Kelly JM (2020) Synthesis and evaluation of novel 2,4-disubstituted arylthiazoles against T. brucei. RSC Med Chem 11(1):72-84

31. Kryshchyshyn A, Kaminskyy D, Karpenko O, Gzella A, Grellier P, Lesyk R (2019 Jul) Thiazolidinone/thiazole based hybrids - New class of antitrypanosomal agents. Eur J Med Chem 174:292-208

32. yu bin, Zhou S, Cao L, Hao Z, Yang D, Guo X et al (2020) Design, Synthesis and Evaluation of Antifungal Activity of Novel Pyrazole-thiazole Carboxamides as Succinate Dehydrogenase Inhibitors. J Agric Food Chem 68:27, 7093-7102

33. Wu Q, Zhao B, Fan Z, Zhao J, Guo X, Yang D et al (2018) Design, synthesis and fungicidal activity of isothiazole-thiazole derivatives. RSC Adv 8(69):39593-39601

34. Sato T, Sekimata K, Sakai N, Watanabe H, Mishima-Tsumagari C, Taguri T et al (2020) Structural Basis of Activin Receptor-Like Kinase 2 (R206H) Inhibition by Bis-heteroaryl Pyrazole-Based 
Inhibitors for the Treatment of Fibrodysplasia Ossificans Progressiva Identified by the Integration of Ligand-Based and Structure-Based Drug Design Appr. ACS Omega. May 26;5(20):11411-23

35. Ebenezer O, Awolade P, Koorbanally N, Singh P (2020 Jan) New library of pyrazole-imidazo[1,2a]pyridine molecular conjugates: Synthesis, antibacterial activity and molecular docking studies. Chem Biol Drug Des 20;95((1):162-173

36. Sivaramakarthikeyan R, Iniyaval S, Saravanan V, Lim W, Mai C, Ramalingan C (2020 May) Molecular Hybrids Integrated with Benzimidazole and Pyrazole Structural Motifs: Design, Synthesis, Biological Evaluation, and Molecular Docking Studies. ACS Omega 5;5((17):10089-10098

37. Xu J, Tan H, Zhang Y, Tang D, Zhan F, Li H et al (2020 Dec) Catalyst-Free One-Pot Synthesis of Densely Substituted Pyrazole-Pyrazines as Anti-Colorectal Cancer Agents. Sci Rep 9;10((1):9281

38. Abdallah M, Gomha S, Abbas I, Kazem M, Alterary S, Mabkhot Y An Efficient Synthesis of Novel Pyrazole-Based Heterocycles as Potential Antitumor Agents. Appl Sci. 2017 Aug 3;7(8):785

39. Secrieru A, O'Neill PM, Cristiano MLS Revisiting the Structure and Chemistry of 3(5)-Substituted Pyrazoles.Molecules. 2019 Dec20;25(1):42

40. Keter FK, Darkwa J Perspective: the potential of pyrazole-based compounds in medicine.BioMetals. 2012 Feb15;25(1):9-21

41. He B, Wu F-X, Yu L, Wu L, Chen Q, Hao G-F et al (2020 May) Discovery of Novel PyrazoleQuinazoline-2,4-dione Hybrids as 4-Hydroxyphenylpyruvate Dioxygenase Inhibitors. J Agric Food Chem 6;68((18):5059-5067

42. Geng W, Jia Y, Chen Y, Ma Q, Fan G, Liao L (2020) Superior thermally robust energetic materials featuring $\mathrm{Z}$ - E isomeric bis(3,4-diamino-1,2,4-triazol-5-yl)-1 H -pyrazole: self-assembly nitrogen-rich tubes and templates with Hofmeister anion capture architecture. CrystEngComm 22(18):3144-3154

43. Lai F, Yu S, Chang Y, Wu T, Wu K, Chang Y et al Comparison study of $\varepsilon$-caprolactone, L-lactide, and $\varepsilon$ decalactone polymerizations using aluminum complexes bearing pyrazole derivatives, and synthesis of polylactide-gradual-poly- $\varepsilon$-caprolactone copolymer.J Polym Sci. 2020 May15;58(10):1400-09

44. Brewer G, Butcher RJ, Zavalij P (2020) Mar Use of Pyrazole Hydrogen Bonding in Tripodal Complexes to Form Self Assembled Homochiral Dimers. Materials (Basel). 31:;13(15957

45. Pedrini A, Maspero A, Bracco S, Comotti A, Galli S, Marchiò L et al (2020) Synthesis, crystal structure, and optical properties of fluorinated poly(pyrazole) ligands and in silico assessment of their affinity for volatile organic compounds. New J Chem 44(16):6443-6455

46. Mahesh K, Ravi K, Rathod PK, Leelavathi P (2020) Convenient synthesis of quinoline-fused triazoloazepine/oxepine derivatives through $\mathrm{Pd}$-catalyzed $\mathrm{C}-\mathrm{H}$ functionalisation of triazoles. New $\mathrm{J}$ Chem 44(6):2367-2373

47. Bharath Kumar Reddy P, Ravi K, Mahesh K, Leelavathi P (2018 Nov) Intramolecular Heck reaction: A facile sequential one-pot synthesis of 1,2,3,4-tetrahydrobenzo[b][1,6]naphthyridines. Tetrahedron Lett 59(45):4039-4043

48. Takate SJ, Shinde AD, Karale BK, Akolkar H, Nawale L, Sarkar D et al (2019 May) Thiazolyl-pyrazole derivatives as potential antimycobacterial agents. Bioorg Med Chem Lett 29(10):1199-1102 
49. Sever B, Altıntop MD, Radwan MO, Özdemir A, Otsuka M, Fujita M et al (2019 Nov) Design, synthesis and biological evaluation of a new series of thiazolyl-pyrazolines as dual EGFR and HER2 inhibitors. Eur J Med Chem 182:111648

50. Vu Quoc T, Tran Thi Thuy D, Dang Thanh T, Phung Ngoc T, Nguyen Thien V, Nguyen Thuy C et al (2019) Some chalcones derived from thiophene-3-carbaldehyde: synthesis and crystal structures. Acta Crystallogr Sect E Crystallogr Commun. Jul 1;75(7):957-63

51. Nagwanshi R, Bakhru M, Jain S Photodimerization of heteroaryl chalcones: comparative antimicrobial activities of chalcones and their photoproducts.Med Chem Res. 2012 Aug29;21(8):1587-96

52. Mujeeb MA, Vedamurthy A, Shettar AK, Puranik SI, Ghagane S, Thimmappa SC In vitro Anti-oxidant and Anti-cancer Activity of Tetradesmus acuminatus Microalgae Extract on MCF-7 Human Breast Cancer Cell Line.Int J Cancer Res. 2020 Jan8;16(1):1-9

53. Kesharwani RK, Kumari S, Singh DB, Tripathi S (2020 Feb) A Docking Simulation Study of Some Herbal Molecules Against Influenza A Virus by Targeting Neuraminidase. Curr Tradit Med 27;6((2):147-154

54. Shiau AK, Barstad D, Loria PM, Cheng L, Kushner PJ, Agard DA et al (1998 Dec) The Structural Basis of Estrogen Receptor/Coactivator Recognition and the Antagonism of This Interaction by Tamoxifen. Cell 95(7):927-937

55. Laskowski RA, Swindells MB (2011 Oct) LigPlot+: Multiple Ligand-Protein Interaction Diagrams for Drug Discovery. J Chem Inf Model 24(10):2778-2786. [cited 2015 Apr 15]; 51(

56. Mishra H, Kesharwani RK, Singh DB, Tripathi S, Dubey SK, Misra K (2019 Dec) Computational simulation of inhibitory effects of curcumin, retinoic acid and their conjugates on GSK-3 beta. Netw Model Anal Heal Informatics Bioinforma 10;8((1):3

\section{Scheme}

Scheme 1 and 2 is available in supplementary section.

\section{Figures}




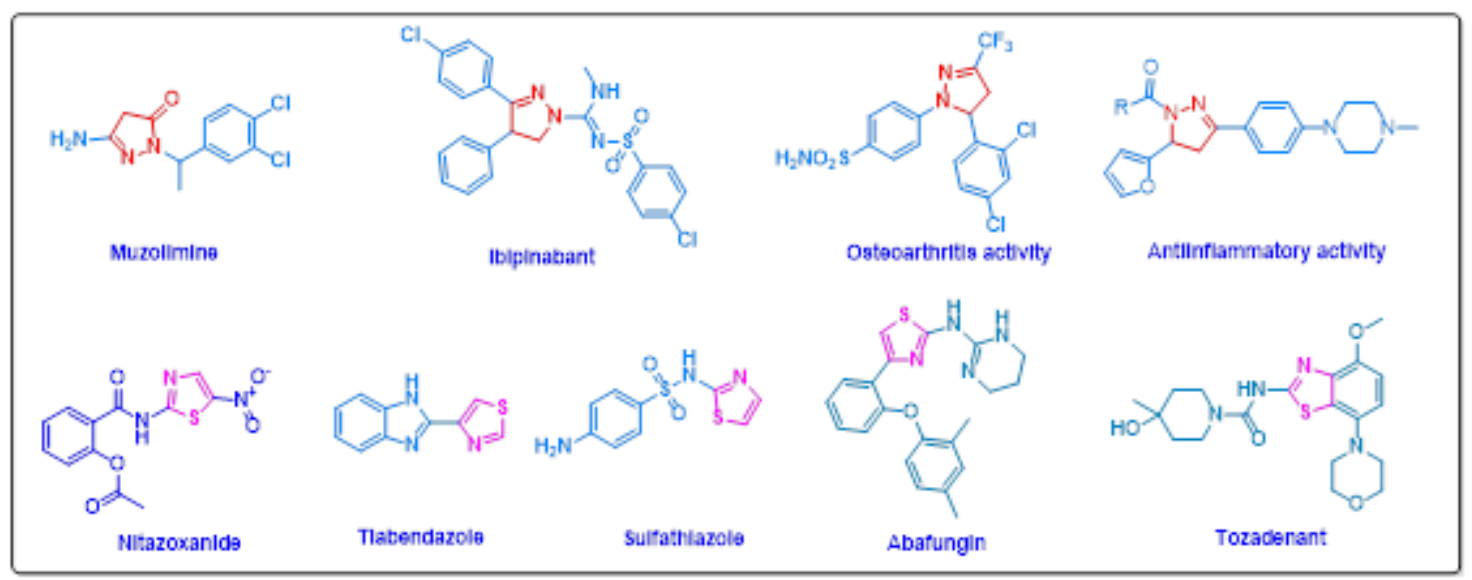

Figure 1

Therapeutic active drugs possessing dihydropyrazole and thiazole scaffolds.

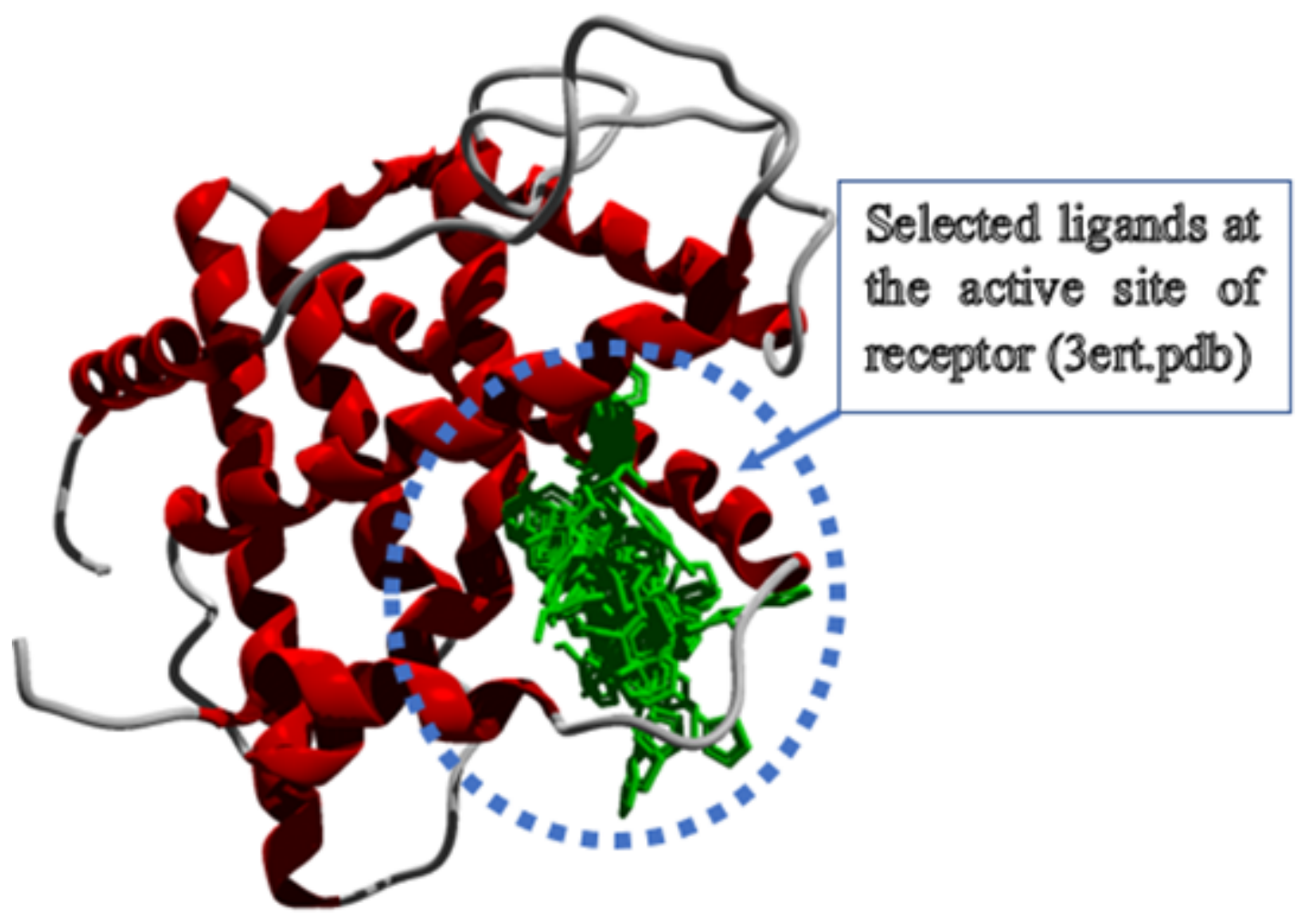

Figure 2

Secondary structure representation of Human Estrogen Alpha protein (3ert.pdb) and bound ligands at the active/binding site cavity. 

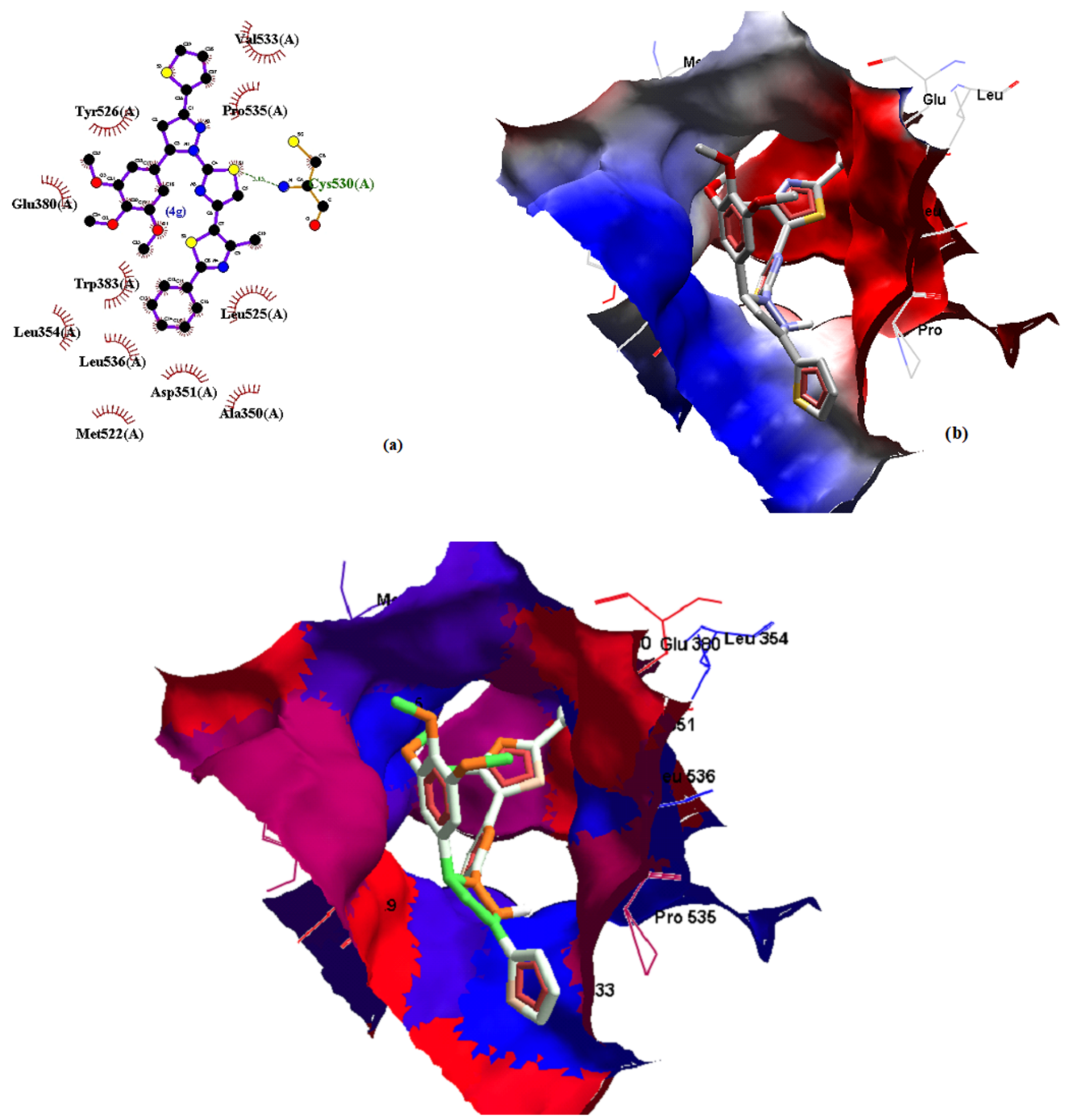

(c)

\section{Figure 3}

(a) 2-D representation of ligand $4 \mathrm{~g}$ with hydrogen bonding view using LigPlus and (b) Electrostatic and (c) hydrophobic interactions at the active site cavity of target protein (3ert.pdb) (Hydrogen bonds- Green color dashed lines; Hydrophobic as an arc ) [55]. 


\section{Supplementary Files}

This is a list of supplementary files associated with this preprint. Click to download.

- Graphical.docx

- Supportinginformation.docx

- Scheme01.png

- Scheme02.png 